\title{
Tubercular Perforation of the Small Bowel: Should All Patients Have an End lleostomy?
}

\author{
Muhammad Imran Aslama, Muhammad Osman Karim ${ }^{\mathrm{b}, \mathrm{d}}$, Syed Hussain Abbas ${ }^{\mathrm{b}}$, \\ Omer Abdalla ${ }^{\mathrm{b}}$, Husnain Shakir ${ }^{\mathrm{c}}$
}

\begin{abstract}
Background: Spontaneous ileal perforation is a common surgical emergency in developing countries due to high incidence of tuberculosis in these countries. Patients diagnosed to have intestinal perforation are universally treated surgically. The aim of this study was to compare the outcome and complication of two procedures usually performed for intestinal perforation i.e., primary repair and ileostomy.

Methods: The study is based on interventional quasi-experimental design with non-probability purposive sampling and was conducted for 6 months between April and October 2010 at a teaching hospital. Sixty patients with intestinal tubercular perforation participated in this study and were divided into two groups. Group A consisted of 30 patients who underwent primary repair and group B consisted of 30 patients who underwent ileostomy. Using Chi-square test, two groups were compared with respect to four outcome variables including duration of hospital stay, complications (peritonitis, wound infection, fever, and obstruction fistula formation) during hospital stay, and complications observed in second week and fourth week follow-ups.
\end{abstract}

Results: The mean age of presentation was $39.13 \pm 18.917$ years (range 13 - 75). Sixty percent of patients were male and $40 \%$ were female. Size of perforation dictated operative decision; in group A, $100 \%$ of patients had a perforation less than $1 \mathrm{~cm}$ and in group B, $100 \%$ of patients had a perforation greater than $1 \mathrm{~cm}(\mathrm{P} \leq 0.05)$. In both groups, $73.4 \%$ of patients had an admission of less than 7 days and $26.6 \%$ of patients stayed beyond this period $(\mathrm{P} \geq 0.05)$. The complication rate in group A during admission was $40 \%$, compared to $30 \%$ in group $\mathrm{B}(\mathrm{P} \geq 0.05)$. In the second postoperative week, com-

Manuscript accepted for publication March 22, 2017

aDepartment of General Surgery, Warwick Hospital, Lakin Road, Warwick, CV34 5BW, UK

${ }^{b}$ Department of General Surgery, Buckinghamshire Healthcare NHS Foundation Trust, Wycombe Hospital, Queen Alexandra Road, High Wycombe, HP11 2TT, UK

'Jinnah Hospital, Usmani Road, Lahore, Pakistan

${ }^{\mathrm{d} C}$ Corresponding Author: Muhammad Osman Karim, Department of General Surgery, Buckinghamshire Healthcare NHS Foundation Trust, Wycombe Hospital, Queen Alexandra Road, High Wycombe, HP11 2TT, UK.

Email: m_osmankarim@hotmail.com

doi: https://doi.org/10.14740/jcs322w plication rate was $16.6 \%$ in group $\mathrm{A}$ and $6.6 \%$ in group $\mathrm{B}(\mathrm{P} \geq 0.05)$. Similarly in the fourth postoperative week, the complication rate was $13.4 \%$ in group $\mathrm{A}$ and $6.6 \%$ in group $\mathrm{B}(\mathrm{P} \geq 0.05)$.

Conclusion: End ileostomy had fewer complications compared to primary closure in management of tubercular small bowel perforation. The choice of surgery was dependent on intra-operative judgement following assessment of the size of perforation and surrounding intra-abdominal contamination. Primary repair may still be a feasible option for perforations less than $1 \mathrm{~cm}$ without gross contamination given similar outcomes.

Keywords: Intestinal tuberculosis; Ileostomy; Primary repair

\section{Introduction}

Spontaneous small bowel perforation is the fifth commonest cause of acute abdominal emergencies in developing countries due to a high incidence of tuberculosis (TB) and enteric fever [1]. In such parts of the world, diagnosis of extra-pulmonary TB poses a challenge due to the broad spectrum of disease, clinical presentation and limited availability of sensitive diagnostic tests. However, in the majority of cases, a process of exclusion facilitates the diagnosis [2]. Primary TB is rare in Western countries. The incidence is however rising due to immigration from developing countries and is common in patients with human immunodeficiency virus infection (HIV) [3, 4].

TB can affect almost any organ of the body and although the most common presentation is pulmonary, extra-pulmonary disease is not rare. The sites involved in extra-pulmonary TB are lymph nodes, abdomen, bones and joints including spine, genitourinary system and central nervous system [5].

Abdominal TB is a chronic inflammatory disorder surpassing Crohn's disease in developing countries with considerable mortality and morbidity $[2,6]$. The diagnosis requires a high index of suspicion due to its vague symptomatology [6]. The histological differential diagnosis of Crohn's disease and intestinal TB can be very challenging, as both are chronic granulomatous disorders with overlapping histological features [7].

Disease manifestation is usually indolent, presenting with late complications including small bowel obstruction (SBO) or 
perforation secondary to tuberculoma mass or stricture formation. The ileocecal region is commonly affected and perforations are usually single and proximal to a stricture $[8,9]$.

There is a paucity of literature regarding operative techniques used in management of such perforations. Here we report outcomes of patients managed according to perforation size and associated intra-abdominal contamination with either primary closure or resection with ileostomy formation.

\section{Materials and Methods}

The study is based on the interventional quasi-experimental design with non-probability purposive sampling and was conducted for 6 months between April 2010 and October 2010 at The Jinnah Tertiary Teaching Hospital, Lahore. The study was registered with the institution and received the necessary ethical approval. All patients who presented as surgical emergency with peritonitis and diagnosed as perforations on clinical basis and met the inclusion criterion were included in the study. Informed consent was taken from all participating patients.

A complete history and examination was performed in all cases and demographic information was also collected. Investigations including ESR, chest X-ray, X-ray abdomen (erect and supine) were performed in all cases. No patient underwent computed tomography as diagnosis was clinical and confirmed on exploratory laparotomy.

The inclusion criteria were patients who presented with a rigid peritonitic abdomen and on exploratory laparotomy were found to have intestinal perforation with intestinal TB confirmed on histopathological analysis. Patients with uncontrolled diabetes, uncontrolled hypertension, previous abdominal surgery and severe malnourishment were excluded from the study. A total of 60 patients who met the inclusion criteria were selected and included in the study.

Patients were allocated into two groups depending on the size of the small bowel perforation. Group A underwent primary repair for small bowel perforations less than $1 \mathrm{~cm}$. Group B underwent bowel resection and ileostomy for small bowel perforations greater than $1 \mathrm{~cm}$.

Primary outcomes assessed postoperatively included duration of admission, pyrexia, wound dehiscence, fistula formation, peritonitis and bowel obstruction. These complications were assessed during the index admission as well as the second and fourth week postoperatively for complications.

Data were analyzed using SSPS version 10.0 software and presented as frequency tables. The two groups were compared using the Chi-square test and a P-value $<0.05$ was considered statistically significant.

\section{Results}

Sixty patients who met the inclusion criteria were included in the study. The mean age of presentation was $39.13 \pm 18.917$ years and median was 35 years (range 13 - 75) with a slight male predominance of $60 \%$ (Table 1 ).

Risk factors of diabetes and/or uncontrolled hypertension
Table 1. Patient Demographics

\begin{tabular}{|c|c|}
\hline & n (\%) \\
\hline \multicolumn{2}{|l|}{ Age (years) } \\
\hline$\leq 30$ & $28(46.7 \%)$ \\
\hline$\geq 30$ & $32(53.3 \%)$ \\
\hline \multicolumn{2}{|l|}{ Sex } \\
\hline Male & $36(60 \%)$ \\
\hline Female & $24(40 \%)$ \\
\hline
\end{tabular}

were observed in $26.6 \%$ of patients in group A compared to $33.4 \%$ of patients in group B ( $\geq 0.05)$. Size of perforation dictated operative decision; in group A, $100 \%$ of patients had a perforation less than $1 \mathrm{~cm}$ and in group B, $100 \%$ of patients had a perforation greater than $1 \mathrm{~cm}(\mathrm{P} \leq 0.05)$. In both groups, $73.4 \%$ of patients had an in-patient admission of less than 7 days and $26.6 \%$ of patients stayed beyond this period $(\mathrm{P} \geq 0.05)$.

The complication rate in group A during the index admission was $40 \%$ with pyrexia evident in $20 \%$, peritonitis in $16.7 \%$ and wound dehiscence in $3.3 \%$ of patients. However, in group B the complication rate was $30 \%$ during the index admission with pyrexia in $16.7 \%$, peritonitis in $10 \%$ and wound dehiscence in $3.3 \%$ of patients. The Chi-square test did not detect a statistically significant difference for complications during the index admission between the two groups ( $\mathrm{P} \geq 0.05)$.

In the second postoperative week, the complication rate in group A was $16.6 \%$ with peritonitis in $10 \%$, wound dehiscence in $3.3 \%$ and fistula formation in $3.3 \%$ of patients. The complication rate in group B was $6.6 \%$ associated with only wound dehiscence. The Chi-square test did not detect a statistically significant difference for complications during the second postoperative week between the two groups $(\mathrm{P} \geq 0.05)$.

In the fourth postoperative week, the complication rate in group A was $13.4 \%$ and was associated with bowel obstruction. The complication rate in group B was $6.6 \%$ and was also associated with obstruction. The Chi-square test did not detect a statistically significant difference for complications during the fourth postoperative week between the two groups ( $\mathrm{P} \geq$ 0.05) (Table 2).

\section{Discussion}

Peritoneal TB is the most common form of abdominal TB and involves alone or in combination with the peritoneal cavity, mesentery and omentum. Abdominal TB can affect any age group but is more common in adolescence. The age of patient presentation in this study ranged from very young (13 years) to very old (75 years). However, the majority were in the age range of 20 - 50 years, which is consistent with other studies $[6,8,10]$. In this study there was a slight male predominance of $60 \%$ contrary to other series reporting more females being affected $[6,8,10,11]$.

Amongst the two groups, $26.6 \%$ of patients in group A had risk factors compared to $33.4 \%$ in group B. Diabetes poses a significant health burden and it is suggested that up to $50 \%$ of 
Table 2. Primary Outcomes

\begin{tabular}{|c|c|c|c|c|}
\hline \multirow{2}{*}{ Primary outcomes } & \multicolumn{2}{|c|}{ Outcome variables, n (\%) } & \multirow{2}{*}{ Pearson Chi-square } & \multirow{2}{*}{ P value } \\
\hline & Group A & Group B & & \\
\hline Size of perforation & & & 60.00 & 0.000 \\
\hline$>1 \mathrm{~cm}$ & $0 / 30(0 \%)$ & $30 / 30(100 \%)$ & & \\
\hline Duration of hospital stay & & & 0.000 & 1.000 \\
\hline$>7$ days & $8 / 30(26.7 \%)$ & $8 / 30(26.7 \%)$ & & \\
\hline Risk factor present & & & 0.317 & 0.573 \\
\hline No risk factor & $22 / 30(73.4 \%)$ & $20 / 30(66.6 \%)$ & & \\
\hline Risk factor (diabetes, hypertension) & $8 / 30(26.6 \%)$ & $10 / 30(33.4 \%)$ & & \\
\hline Complication during hospital stay & & & 0.659 & 0.417 \\
\hline \multirow{2}{*}{ Complication in second week follow-up } & $25 / 30(76.7 \%)$ & $28 / 30(93.3 \%)$ & & \\
\hline & $5 / 30(16.6 \%)$ & $2 / 30(6.6 \%)$ & & \\
\hline \multirow[t]{3}{*}{ Complication in fourth week follow-up } & & & 0.7407 & 0.389 \\
\hline & $26 / 30(86.6 \%)$ & $28 / 30(93.4 \%)$ & & \\
\hline & $4 / 30(13.3 \%)$ & $2 / 30(6.6 \%)$ & & \\
\hline
\end{tabular}

the patients with diabetes will require surgery at some point in their lives [12]. Reported surgical mortality in diabetics is 1.5 times higher compared to non-diabetics [13].

The duration of index admission was the same in both groups with $73.4 \%$ of patients discharged within 7 days. However, when compared to reported series literature suggests primary closure is associated with shorter length of hospital stay $[14,15]$.

Complications were evident in both groups during the index admission. Group A had a higher complication rate of $40 \%$ compared to $30 \%$ in group B and was mainly associated with pyrexia and localized peritonitis secondary to anastomotic leak. In cases where this did occur, operative resuscitative management with intravenous fluids and antibiotics was initiated. Anastomotic leak is a serious complication of gastrointestinal surgery and is associated with a significant morbidity and mortality [16]. Only 3.3\% cases in group A developed wound dehiscence reflecting sequel of other complications described [17]. In the immediate period, group B had better outcomes with fewer cases of localized peritonitis.

In the second postoperative week, the complication rate in group A was $16.6 \%$ compared to $6.6 \%$ in group B and was secondary to persistent localized peritonitis with associated wound dehiscence and fistula formation. However, patients only suffered from minor wound dehiscence. These findings are consistent with current evidence regarding anastomotic leaks that suggests they may occur late in the postoperative period and even after hospital discharge [14]. This therefore highlights the importance of prospective data collection and adequate follow-up [14]. In group B, only wound dehiscence was seen in $6.7 \%$ cases which suggests resection of the affected segment and end ileostomy is a safer option in treating the tuberculous intestinal perforation.

Overall, surgeons are all too familiar with the potentially devastating consequences of an anastomotic leak [14]. This is evident in the fourth postoperative week, where in group A $13.3 \%$ of patients developed sub-acute SBO which was successfully managed conservatively. It was, in part, due to the anastomotic leak and adhesions formed between intestinal loops. The patients were managed without fecal diversion, as the leak was small and the obstruction was partial. Compared with other series, patients with small contained leaks, which tend to present later in the clinical course and can often be treated without fecal diversion [15] were less prevalent in group B with only $6.7 \%$ of patients being affected.

In group B only $6.7 \%$ patients presented with partial obstruction of the stoma which were managed conservatively with stomal dilatation. The findings suggest that end ileostomy appears to be a preferential management option in those with tubercular perforation of the small bowel.

Overall, patients with small bowel perforations less than $1 \mathrm{~cm}$ underwent primary closure and those with perforations greater than $1 \mathrm{~cm}$ required resection and ileostomy. Formation of ileostomy for the tubercular perforation had slightly better outcomes although not statistically significant. The limitations of this study include small sample size as well as heterogeneity of the patient cohort which make it difficult to comment on cause-effect relationships and detect a difference. Further 
research would therefore involve multi-center collaboration.

\section{Conclusion}

End ileostomy had fewer complications compared with primary closure in management of tubercular small bowel perforation. However, the choice of surgery was dependent on intra-operative judgement following assessment of the size of perforation and surrounding intra-abdominal contamination. However, primary repair may still be a feasible option for perforations less than $1 \mathrm{~cm}$ without gross contamination given similar outcomes.

\section{Conflicts of Interest}

The authors declare that they have no conflicts of interest.

\section{Funding}

None.

\section{Disclosure}

The authors have nothing to disclose.

\section{References}

1. Rehman A. Spontaneous ileal perforation. Journal of Postgraduate Medical Institute. 2003;17:105-110.

2. Chana GA, Khan MA. Abdominal tuberculosis "Surgeons Perspective". Journal of Surgery of Pakistan. 2003;8:4-9.

3. Sefr R, Rotterova P, Konecny J. Perforation peritonitis in primary intestinal tuberculosis. Dig Surg. 2001;18(6):475479.

4. Ara C, Sogutlu G, Yildiz R, Kocak O, Isik B, Yilmaz S, et al. Spontaneous small bowel perforations due to intestinal tuberculosis should not be repaired by simple closure. J GastrointestSurg. 2005;102:31-32.
5. Karima MM, Chowdhuryb SA, Hussainc MM, Faizd MA. A Clinical Study on Extrapulmonary Tuberculosis. Journal of Bangladesh College of Physicians and Surgeons. 2006;24(1):19-28.

6. Wadhwa N, Agarwal S, Mishra K. Reappraisal of abdominal tuberculosis. J Indian Med Assoc. 2004;102(1):3132.

7. Kirsch R, Pentecost M, Hall Pde M, Epstein DP, Watermeyer G, Friederich PW. Role of colonoscopic biopsy in distinguishing between Crohn's disease and intestinal tuberculosis. J Clin Pathol. 2006;59(8):840-844.

8. Ahmed M, Mainghal MA. Pattern of mechanical.Intestinal Obstruction in adults. J Coll Physicians Surg Pak. 1999;9:441-443.

9. Kapoor VK. Abdominal tuberculosis. Postgrad Med J. 1998;74(874):459-467.

10. Muhammad SaleemShaikh, Kheo Ram Dholia, Mohammad AyoubJalbani, Sikandar Ali Shaikh. Prevalence of intestinal tuberculosis in cases of acute abdomen. Pakistan Journal of Surgery. 2007;23(1):52-56.

11. Naz F, Chaudhry ZA, et al. Abdominal Tuberculosis: A review of 25 cases. Ann King Edward Med Coll. 1999;5:180-183.

12. Reynolds C. Management of the diabetic surgical patient. A systematic but flexible plan is the key. Postgrad Med. 1985;77(1):265-269, 272-266, 279.

13. Milaskiewicz RM, Hall GM. Diabetes and anaesthesia: the past decade. Br J Anaesth. 1992;68(2):198-206.

14. Neil Hyman, Thomas L. Manchester, Turner Osler, Betsy Burns, Peter A. Cataldo. Anastomotic Leaks After Intestinal Anastomosis; It's Later Than You Think. Ann Surg. 2007;245(2):254-258.

15. Alves A, Panis Y, Pocard M, Regimbeau JM, Valleur P. Management of anastomotic leakage after nondiverted large bowel resection. J Am Coll Surg. 1999;189(6):554559.

16. Anis Subhan, Nusrat Anis, Abdul Majeed Baloch. One Layer Interrupted Intestinal Anastomosis. J Surg Pak Jun. 2001;6(2):9-10.

17. Waqar SH, Malik ZI, Razzaq A, Abdullah MT, Shaima A, Zahid MA. Frequency and risk factors for wound dehiscence/burst abdomen in midline laparotomies. J Ayub Med Coll Abbottabad. 2005;17(4):70-73. 